\title{
Nanometer positioning accuracy over a long term traveling stage based on heterodyne interferometry
}

\author{
I. Naeim and S. Khodier \\ Length Metrology, NIS, Egypt
}

Received: 5 May 2012 / Accepted: 14 June 2012

\begin{abstract}
In order to achieve nanometer accuracy, metrologists need to identify the sources of error and develop solutions to eradicate or minimize their effects. A stabilized low power dual-frequency laser heterodyne interferometer (ZMI-1000A) designed to measure linear and angular displacement with nominal measurement resolution $1.24 \mathrm{~nm}$ and 0.0025 arcsec, respectively, is used to achieve measurement of displacements over different travelling axes by comparison with the electronically reference measurements of the stage over wide range $500 \mathrm{~mm}$. The repeatability and reversal error of linear stage over the working distance have agreed opto-electronically and the positioning uncertainty been reduced. A multivariable framework was implemented for the $x$-axis due to the cross coupling between the forward and backward course of the linear stage. Thermal error reduction is achieved using environmental temperature control $\left(20 \pm 0.2{ }^{\circ} \mathrm{C}\right)$ to help reduce thermal errors.
\end{abstract}

Keywords: Laser heterodyne interferometer; displacement metrology

\section{Introduction}

Displacement related measuring techniques play an important role in current modern technology. Nanometer level accuracy in displacement metrology applications has seen an increase in demands recently [1]. Developing high precision interferometric stages with long traveling range is definitively crucial for the development of nanotechnology. Aside from high resolution and system stability, large area positioning is also an important factor for nanotechnology applications. In general, high precision interferometric stages consist of two parts; a measurement device and a driving device as shown below in Figure 1. The resolution of these measurement devices can reach the nanometric level and even the sub-nanometric level. Heterodyne interferometry is inherently more accurate than standard interferometry because it is less sensitive to intensity noise $[2,3]$.

The utilization of a precise and traceable wavelength, together with a stable reference wavefront makes our laser heterodyne interferometer useful as an absolute measurement device may easily calibrate any movable machinery part. Current technology on the accuracy calibration of linear stages, CMMs, and machine tools frequently employs the laser interferometer in association with autocollimator and electro-mechanical tools [4]. The actual traveling path and orientation of the stage (Newport, IMS-LM500) may deviate from the straight line of motion due to its own geometric errors. As shown in Figures 1-4 a moving carriage/stage along a specified axis will inherently have estimated geometric errors over its working area.

\section{Theoretical basis}

As opposed to standard interferometry, where the optical path difference OPD information is encoded on the intensity of an interference pattern, heterodyne interferometry encodes the OPD on the phase of an oscillating interference pattern. The optical phase difference $\Delta \phi$ between the object and reference beam is encoded as the phase of the amplitude modulated output signal Imes, which at first, has a frequency equal to the frequency difference $\Delta f$ introduced by the internal frequency shifter. Mathematically, we can express this as:

$$
\begin{gathered}
U_{\text {ref }}=A_{1} e^{i 2 \pi f r t} \\
U_{\text {disp }}=A_{2} e^{i 2 \pi f m t+\Delta \phi_{\mathrm{OPD}}} \\
I_{\mathrm{mes}}=\left(U_{\text {ref }}+U_{\mathrm{disp}}\right)\left(U_{\mathrm{ref}}+U_{\mathrm{disp}}\right)^{*} \\
I_{\mathrm{mes}}=A_{12}+A_{22}+A_{1} A_{2} \cos \left(2 \pi \Delta f t \pm \Delta \phi_{\mathrm{OPD}}\right) \\
\Delta \phi_{\mathrm{OPD}}=2 \pi\left(L_{\mathrm{OPD}} / \lambda_{\mathrm{m}}\right) .
\end{gathered}
$$




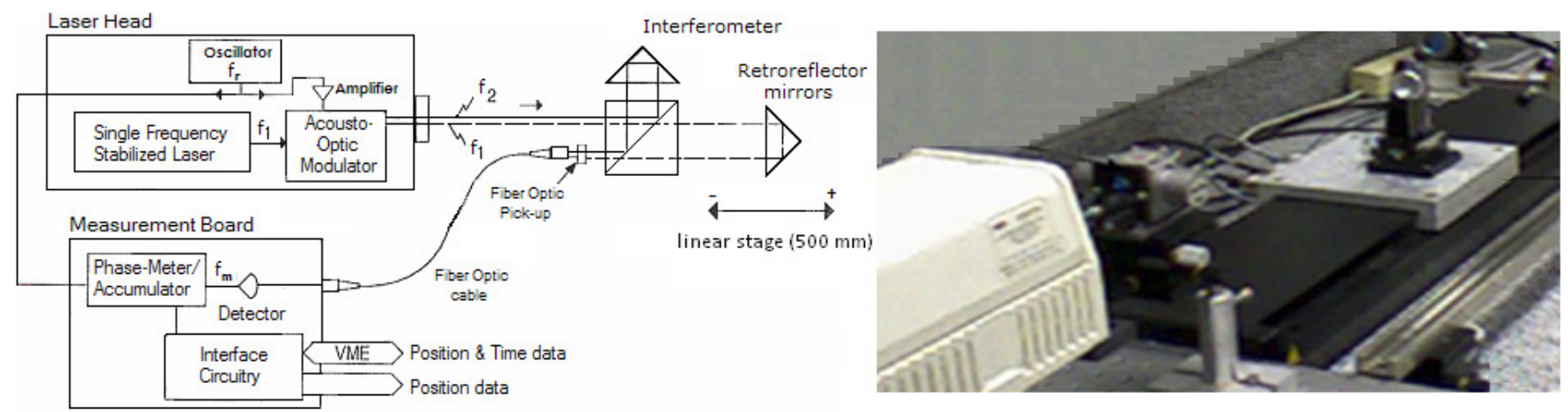

Fig. 1. Schematic diagram and photo of the proposed experimental setup.

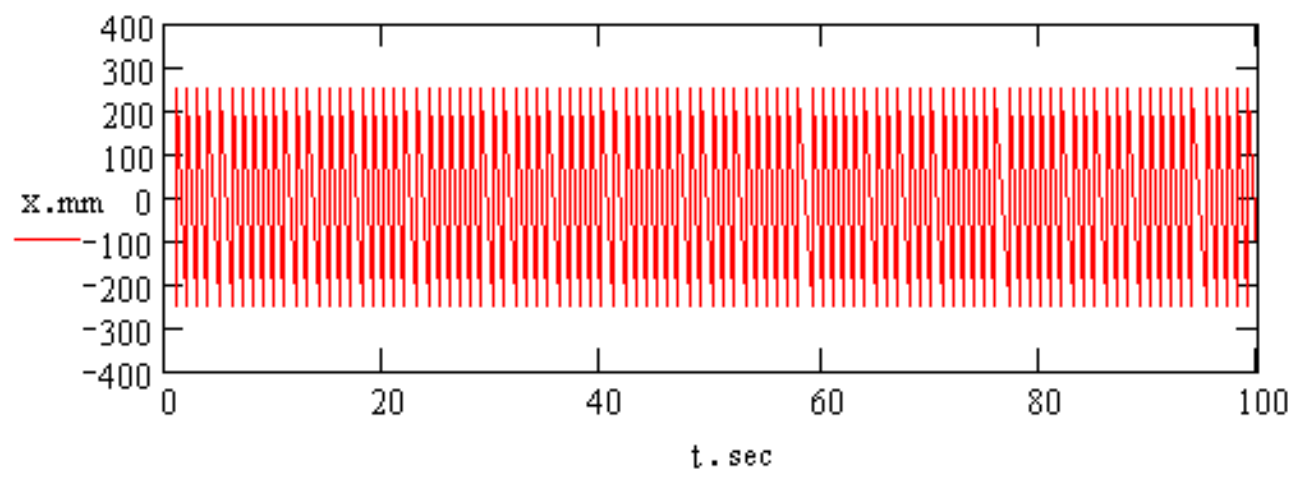

Fig. 2. Stepping linear displacement $500 \mathrm{~mm}$ over $100 \mathrm{sec}$ incremental times.

The resultant optical phase difference is due to the pathlength difference between the reference and object beams on the displacement stage. The proposed system which consists of two main parts (heterodyne interferometer and electrically controlled traveling stage) can successfully measure positioning deviation over the long range motion $500 \mathrm{~mm}$ displacement (forward/backward) with high resolution $5 \mathrm{~nm}$ where the minimum linear incremental motion $20 \mathrm{~nm}$; misalignment angle ideally equal null which induce a cosine error on the displacement $\varepsilon=D \times \theta^{2} / 2$. The proposed system actually cannot be used to measure two degree of freedom straightness simultaneously because of the non common optical path configuration of the designed system. All the displacements reported in this paper have been obtained using a Zygo interferometric system with a controlled programmable Newport stage.

Over multi-axes motion system, a fabricated error of the motion system will result in an error motion which appears to be orthogonal to the direction of the proposed moving path. This error is known as a straightness error and needs to be measured or compensated for. In general, the interferometers that measure the movement parallel to the measurement axis are called linear interferometers [5]. In order to know what is the effect of using different velocities in displacement courses that may apply in advanced technology. A multivariable framework was implemented over the $x$-axis due to the cross coupling between the forward and backward course of the linear stage.

\section{Principle of measurement}

In order to fully map the errors of the $X Y$ high precision linear stage and apply proper error compensation. The error measurements were performed using heterodyne laser interferometry with different optics for each type of error. The concept of a measurement system requires that values assigned to represent certain characteristics of objects be reasonably unique and repeatable over time and changes in location.

Sequences of measurements of the same thing made at various times and at different locations show evidence of convergence to the same limiting mean, Figure 2.

In order to illustrate the nature of a measurement process, consider first the collection of simulated measurementdvips in Figure 2. The data shown reflects the effects of variability from both cyclic and step sources over time. For the 500 measurements, the data has the appearance of coming from a reasonably well behaved measurement process. The measurements tend to cluster around the central line.

Confidence that the process has a single limiting mean is strengthened. With process performance as shown, a predictive statement concerning the next can be considered. Uncertainty statements are, in essence, predictors of the degree to which such agreement can be attained. Failure to agree within uncertainty limits is an indication that the two processes are fundamentally different, 

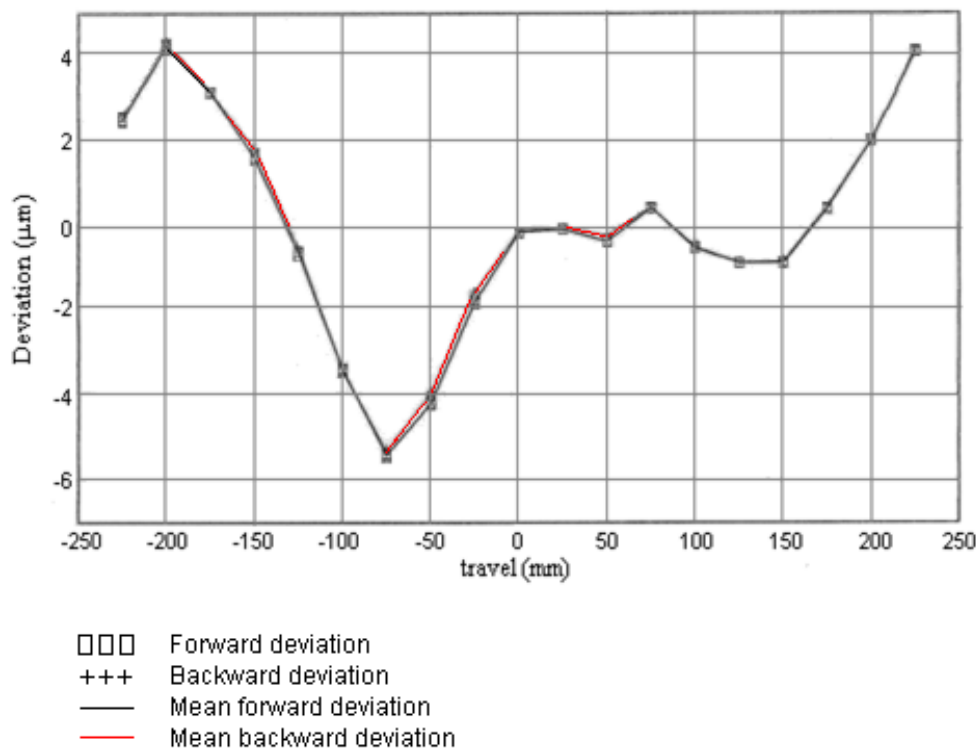

Fig. 3. Geometric errors measurement.

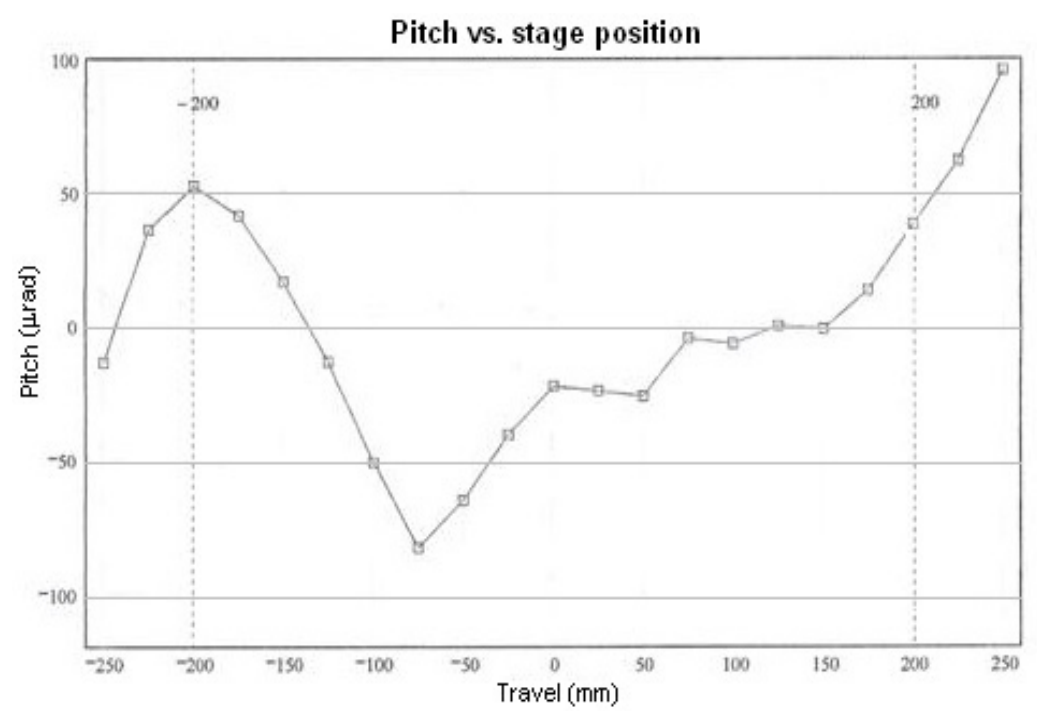

Fig. 4. Pitch vs. stage position over the $500 \mathrm{~mm}$ traveling-path.

or that the uncertainty statement does not adequately describe the error bounds.

It is important to know the bounds for the variability which occurs in the course of making measurements. Redundancy, either by repeated measurements or incorporated in a particular measurement process, provides a means for assessing this variability [6].

\section{Experimental measurement and results}

A schematic of the experimental setup is shown in Figure 1. Accuracy, reversal error, and repeatability over the long traveling pass on $x$-axis $(-250 \mathrm{~mm} \leftrightarrow+250 \mathrm{~mm})$ forward and backward using pendulum step method. As shown in Figure 3, the obtained results: the positioning accuracy: (peak to peak) $9.64 \mu \mathrm{m}$, and slope linear
$-2 \times 103 \mu \mathrm{m} / \mathrm{mm}$. Reversal error B (hysteresis): B (peak to peak) $248 \mathrm{~nm}$ and B (standard deviation) $76.74 \mathrm{~nm}$. Repeatability R (Peak to peak) is $135 \mathrm{~nm}$ and R (standard deviation) is $47.53 \mathrm{~nm}$. As shown in figure below, over the first $50 \mathrm{~mm}$ (from 0 to 50 ), the positioning error is approximately zero. However, within this motion range the accuracy can be maintained. All the system elements are secured on an optical table to avoid any vibration which may affect the accuracy of the results. Measurements were performed by moving the linear stage for a travel of $450 \mathrm{~mm}$ with an increment of $5 \mathrm{~mm}$, for a number of three runs.

This paper proposes a simple and easily implemented system for the simultaneous measurement of pitch and yaw of the moving axes of a linear stage using different techniques. As shown in Figure 4, over the $500 \mathrm{~mm}$ 
traveling path, Pitch and Yaw measurements: (pitch $\theta x$ verses stage position) on the travel $178.7 \mu \mathrm{rad}$, and (yaw $\theta y$ verses stage position) on the travel $84 \mu \mathrm{rad}$. The proposed measuring system is a useful geometric error measurement system; it can be also integrated to $x y z$ degreeof freedom stage for the precise motion control. Initial straightness measurements were made before final ZMI alignment; data were taken at $1 \mathrm{~mm}$ increments over the full $500 \mathrm{~mm}$ stage travel. The sub-nm repeatability ripple with a spatial and temporal period has been achieved optoelectronically.

\section{Calibration using optical interferometry}

Displacement measurements by optical interferometry depend on the induced phase difference and on the interferometer's sensitivity vector; the latter depends in turn on the illuminating sources and on the geometry of the optical arrangement. We have performed an uncertainty analysis of the in-plane displacements measured by heterodyne interferometry. Each axis of a plan mirror interferometer has three associated measurement vectors; the interferometer beam, mirror normal, and axis of measurement. Since the laser interferometer system counts the number of wavelengths of distance traveled, actual displacement can be determined $[7,8]$. As shown in Figure 1, the phase-meter is an electronic device designed to accumulate and measure the phase shift of the measurement electrical signal to the reference electrical signal. Interface circuitry converts the phase information into measurement data. i.e., the function of the measurement board in the heterodyne DMI (Displacement Measuring Interferometer) is to convert a measurement signal from an interferometer and a reference signal from laser head into measurement data [9].

Displacement $=($ No. counts

$\times$ wavelength/resolution factor).

The equation used in our system that depending on the interferometer type, resolution, and the wavelength of the measurement beam is $D=N \lambda / 256$. Traceability is ensured by using a stabilized laser that produces a calibrated and therefore traceable wavelength.

Aside from high resolution and system stability, large area positioning is also an important factor for nanotechnology applications. However, once the measurement system is built, it is natural for vibrations to still occur. The drive system, with advanced control algorithms, can be used to actively dampen the vibrations. The obtained results are decrease for the same displacement with increasing the number of repetitions.

Experimental results show an expanded uncertainty of about $0.7 \mathrm{~nm}+0.1 \times 10^{-3} \mathrm{~L}$. The calibrated actuator is then used as a transfer standard at the nanometer level, both for testing the nonlinearity of the laser interferometer and for calibrating the linear variable differential transformer probe (Line Standards) of the National Institute of Standards (Egypt), [10-12].

\section{Conclusion}

In this paper, metrology tests were performed to measure geometric errors of the linear stage over $x$-axis in two directions. Calibrations are performed using heterodyne laser interferometer in accordance with international metrology standards. The setup for each test is outlined in Sections 4 and 5 with an explanation, and how it contributes to measured errors. Section 4 focuses on the linear positional error and presents the angular errors for each axis, while Section 5 examines straightness errors optoelectronically. Each test is performed at three locations along each axis directions to produce an actual error map of the $X Y$ linear stage. Finally, an overall error budget is presented with focus on the geometric and dynamic errors, where the maximum static resultant error of the $X Y$ stage was estimated to be $144 \mathrm{~nm}$, and the maximum dynamic resultant error of $369 \mathrm{~nm}$.

Acknowledgements. We would like to thank Prof. M. Bahrawi for his scientific support.

\section{References}

1. A.S. Dennis, Length, dimensional measurements at NIST, J. Res. Natl. Inst. Stan. 106, 1-23 (2001)

2. P.S. Huang, J. Ni, On-line error compensation of coordinate measuring machines, Int. J. Mach. Tools Manuf. 35, 725-738 (1995)

3. K.C. Fan, M.J. Chen, W.M. Huang, A six-degree-offreedom measurement system for the motion accuracy of linear stage, Int. J. Mach. Tools Manuf. 38, 155-164 (1998)

4. Shigeru Hosoe, High precise and stable displacementmeasuring laser interferometer with differential optical paths, Precis. Eng. 17, 258-265 (1995)

5. Noh-Bin Yim, Cheon II Eom, Seung-Woo kim, Dual-mode phase measurement for optical heterodyne interferometery, Meas. Sci. Technol. 11, 1131-1137 (2000)

6. S. Wetzels, Laser based displacement calibration with nanometer accuracy, Ph.D. thesis, TUE, Eindhoven, The Netherlands, 1998

7. F.C. Demarest, High-resolution, high-speed, low data age uncertainty, heterodyne displacement measuring interferometer electronics, Meas. Sci. Technol. 9, 1024-1030 (1998)

8. ZMI-1000A Operating manual, ZYGO Corporation, Middlefield, 1999

9. S.G. Rabinovich, Measurement Errors and Uncertainties, 3rd edn. (Springer, New York, 2005)

10. M. Bahrawi, N. Fareed, Application of a commercially available displacement measuring interferometer to line scale measurement and uncertainty of measurement, Mapan-JMSI 25, 261-266 (2010)

11. A. Sacconi, G.B. Picotto, W. Pasin, The IMGC calibration setup for micro-displacement actuators, IEEE Trans. Instrum. Meas. 48, 483-487 (1999)

12. J.M. Gorniak, Design and Metrology of a Precision XY Planar Stage, Master of Applied Science in Mechanical Eng., Waterloo, Ontario, Canada, 2010 